\title{
Characterization and prognosis of patients with hepatocellular carcinoma (HCC) in the non-cirrhotic liver
}

Kerstin Schütte ${ }^{1}$, Christian Schulz ${ }^{1}$, Janine Poranzke ${ }^{1}$, Kai Antweiler ${ }^{2}$, Jan Bornschein ${ }^{1}$, Tina Bretschneider ${ }^{3}$, Jörg Arend ${ }^{4}$, Jens Ricke $^{3}$ and Peter Malfertheiner ${ }^{1 *}$

\begin{abstract}
Background: HCC predominantly develops in the condition of chronic inflammation that has led to liver cirrhosis. A small proportion of patients with HCC is diagnosed in the non-cirrhotic liver (NCL). Data on patients with HCC in $\mathrm{NCL}$ in advanced stages are scarce.

Methods: A retrospective analysis was performed comparing 93 patients with HCC in NCL to 571 patients with HCC in liver cirrhosis (LC) with respect to clinical and demographic characteristics. Also factors influencing survival in patients with HCC in NCL were analyzed.

Results: Patients with HCC in NCL were diagnosed at older age and in more advanced tumor stages than patients with LC. More than $25 \%$ of patients with HCC in NCL presented with extrahepatic metastases. Only a minority of patients with HCC in NCL lacked any sign of hepatic damage. Risk factors for LC and risk factors for NAFLD are present in the majority of patients with HCC in NCL. The BCLC classification corresponded with the survival of patients with HCC in NCL although the therapeutic options differ from those for patients with liver cirrhosis.

Conclusions: It will be one of the major challenges in the future to awake awareness of carrying a risk of hepatic malignancies in patients with chronic liver diseases apart from liver cirrhosis, especially in NAFLD. Surveillance programs need to be implemented if these are cost-effective.
\end{abstract}

Keywords: Hepatocellular carcinoma, Prognosis, Liver cirrhosis, Non-cirrhotic liver, NAFLD

\section{Background}

The incidence of hepatocellular carcinoma (HCC) is constantly rising throughout the world with the majority of cases in Asia and Africa due to the high prevalence of hepatitis B virus (HBV) infection [1]. Although Europe is still considered to be a low incidence area, the incidence of HCC in Germany has risen [2] to 6.2 cases/100 000/ year with a high mortality of 5.2/100 000/year [3]. HCC develops predominantly in the condition of chronic inflammation evolving into liver cirrhosis (LC) [2]. Therefore liver function in addition to tumor stage and patient

\footnotetext{
* Correspondence: peter.malfertheiner@med.ovgu.de

'Department of Gastroenterology, Hepatology and Infectious Diseases, Otto-von-Guericke University Magdeburg, Leipziger Str. 44, Magdeburg 39120, Germany

Full list of author information is available at the end of the article
}

related factors has a major impact on treatment decision and prognosis in case of $\mathrm{HCC}$.

The proportion of patients with HCC diagnosed in a non-cirrhotic liver (NCL) varies throughout different geographic regions of the world ranging from $7 \%$ to $54 \%$ and depends strongly on the leading risk factor for hepatocarcinogenesis [4]. In Western countries, $15 \%-20 \%$ of HCCs are diagnosed in the absence of LC $[1,5,6]$. Most reports on these patients are from surgically treated cohorts of patients that have undergone curative resection with an obvious selection bias. Data on patients with HCC in NCL in more advanced stages with respect to clinical features and factors influencing survival are scarce in Europe.

Strikingly, previous studies reveal a lower male preponderance of HCC in NCL than in LC. The three main risk factors for $\mathrm{HCC}$ ( $\mathrm{HBV}$ or $\mathrm{HCV}$ infection and alcohol 
abuse) are less frequent than in patients with LC. Patients with HCC in NCL present at more advanced tumor stages than patients with HCC in LC $[4,7]$ because tumors are generally detected when the disease has become symptomatic. The reason for this is that HCC in LC are frequently detected during surveillance ultrasound. However, a larger proportion of patients with HCC in NCL can be treated with curative intent because hepatic resections without the risk of postoperative liver failure are more likely. The absence of advanced underlying chronic liver disease leads to the fact that the tumor burden is the most significant factor influencing survival among further tumor-related, demographic and etiological factors $[4,5]$.

\section{Methods}

Aiming at a clinical characterization of patients with HCC in NCL, a retrospective analysis in a large singlecenter cohort in Germany was performed. Patients with HCC in NCL were compared to patients with HCC in LC with respect to demographic and clinical characteristics. Additionally, factors influencing the survival of patients with HCC in NCL were analyzed.

The medical records of 714 patients diagnosed and treated with HCC at the University Hospital of Magdeburg between February 1994 and January 2013 were analyzed retrospectively. The analysis included patients who were referred to the Department of Gastroenterology, Hepatology and Infectious Diseases, to the Department of Surgery or to the Department of Radiology and Nuclear Medicine. After excluding patients in whom sufficient data was not available for the purpose of this study, 664 patients were included into the final analysis. Of these, 571 were diagnosed with HCC in LC and 93 patients (14.01\%) suffered from HCC in a non-cirrhotic liver.

The retrospective analysis was performed following the guidelines of the Declaration of Helsinki and approved by the ethical review committee of the Otto-vonGuericke University Magdeburg, Germany.

\section{Epidemiological data}

Age, gender, height, weight, calculation of body mass index (BMI) and performance status at the time of diagnosis were recorded.

Liver cirrhosis was either diagnosed histologically or by typical clinical signs, i.e. findings consistent with portal hypertension (enlarged spleen, ascites, esophageal or gastric varices or portal hypertensive gastropathy) combined with sonographical findings. The diagnosis of a non-cirrhotic liver was based on reports of the histopathological evaluation of liver tissue obtained by surgery at time of the resection of HCC or obtained by biopsy from the non-tumor-tissue at the time of diagnosis of HCC. Patients without histological sampling were classified as non-cirrhotic if they were completely free of any evidence of cirrhosis based on clinical, laboratory and radiological findings.

Liver cirrhosis was considered to be a consequence of chronic alcohol abuse if the patient had reported a consumption of more than $60 \mathrm{~g}$ alcohol/day or if a history of chronic alcohol abuse was documented in the past medical history. Data on the status of hepatitis B viral infection was available for all and on hepatitis $C$ virus infection for 643 patients.

Information on prevalence of diabetes mellitus as a metabolic risk factor was available for 427 patients. NAFLD was diagnosed by criteria for steatosis in transabdominal ultrasound in the absence of other possible causes of liver disease.

\section{Serum parameters}

To diligently characterize underlying chronic liver disease with respect to liver function, inflammatory activity and etiology, a panel of parameters on clinical chemistry were extracted from the medical records. These included levels of bilirubin, albumin, quick, prothrombin time, alkaline phosphatase, gamma-glutamyl transferase, alanine aminotransferase, aspartate aminotransferase, creatinine, hemoglobin, platelet count, urea nitrogen and protein. If available, levels of ferritin, transferrin saturation, immunoglobulin (Ig) G, IgA, IgM, coeruloplas$\mathrm{min}$, antinuclear antibodies, antibodies against soluble liver antigen, anti-liver kidney microsomal antigen and alpha-1-antitrypsin were analyzed, too. Additionally, the level of alpha-fetoprotein (AFP) at the time of diagnosis was evaluated. All serum parameter values were classified according to a specified threshold for explorative analyses on parameters' impact on survival.

\section{Tumor stage}

Radiological reports were used for the assessment of tumor stage. Number and size of HCC nodules as well as the presence of distant metastases including lymph node involvement and information on the patency of the portal vein were recorded. The Barcelona-Clinic Liver Cancer (BCLC) stage was defined respecting tumor stage, liver function as assessed by Child-Pugh-score in patients with LC and clinical performance status of the patient [8]. Additionally, the CLIP score was rated for all patients if possible $[9,10]$.

\section{Survival data}

In 397 cases the exact date of death was taken from the medical records. For the remaining patients, data on survival was censored at the time of the last documented contact to the patient. Thus, the length of survival was calculated from the date of HCC diagnosis to the date of death or to the date of the last documented contact, respectively. 


\section{Statistical analysis}

All statistical analyses were performed using IBM SPSS Statistics 21.0.0 (IBM Corporation, New York, N.Y., USA). Results for numerical data are given as median together with minimum and maximum of the sample (i.e. range). For categorical data, results are given as absolute numbers with percentage. For comparison of categorical data, Chi-Square-tests were applied. MannWhitney U-tests were used for testing homogeneity of independent samples in continuous data. For the analysis of individual factors influencing survival Cox regression analyses were performed. Factors that were shown to have a significant input on patients' survival at univariate analysis were included into a multivariate Cox regression analysis except for the two staging systems (CLIP score and BCLC score) that are influenced by several of the clinical and tumor related factors. Median survival times that were also compared by log-rank tests and Kaplan-Meier curves are given for single influencing factors. All tests were carried out two-sided. The level of significance was set to 0.05 without adjusting for multiplicity. The analysis has to be regarded as explorative.

\section{Results}

The demographic characteristics of patients analyzed are summarized in Table 1. Patients with LC and HCC were diagnosed at a median age of 66 years (range 31-85 years) while patients with HCC in NCL were diagnosed at a statistically significant older age (median age 69 years (range 32-85 years), $\mathrm{p}=0.004$ ) (Figure 1).
Of 571 patients with $\mathrm{HCC}$ in LC, 103 were female, resulting in a male to female ratio of $4.5: 1$ while the proportion of female patients was larger in patients with HCC in NCL with a male to female ratio of $2.7: 1$ ( $p=0.045)$. Female patients were diagnosed with $\mathrm{HCC}$ in NCL at a median age of 66 years while male patients presented at a median age of 71 years $(\mathrm{p}=0.383)$. No statistically significant differences between patients with HCC in NCL and patients with $\mathrm{HCC}$ in LC were depicted with respect to their clinical performance status at the time of diagnosis.

When analyzing potential underlying liver diseases or risk factors for the development of HCC, significant differences between the two cohorts were apparent. While in a significant proportion of patients with $\mathrm{LC}$ alcohol was identified as causative factor, alcoholic liver disease played a minor role in patients with HCC in NCL and was identified in $15.05 \%$ of patients. Viral hepatitis was diagnosed in $7.53 \%$ of patients with HCC in NCL (HCV $\mathrm{n}=3$; HBV $\mathrm{n}=4)$. NAFLD was diagnosed in 6 patients (6.5\%). Of these 6 patients, 5 suffered from diabetes mellitus, 5 had been diagnosed with arterial hypertension and 4 presented with a $\mathrm{BMI}>25 \mathrm{~kg} / \mathrm{m}^{2}$. With respect to all patients with $\mathrm{HCC}$ in NCL, the BMI exceeded $25 \mathrm{~kg} /$ $\mathrm{m}^{2}$ in $31(33.3 \%)$ patients, 8 patients $(8.6 \%)$ were obese and presented with a BMI exceeding $30 \mathrm{~kg} / \mathrm{m}^{2}$. A significant proportion of patients with HCC in NCL had been diagnosed with diabetes mellitus ( $n=42,45.16 \%$ ), in 27 patients (29\%) a clinical diagnosis of steatosis of the liver had been established before. In the majority of patients $(\mathrm{n}=53 ; 56.99 \%)$ with $\mathrm{HCC}$ in NCL no underlying

Table 1 Patients' demographic characteristics

\begin{tabular}{|c|c|c|c|c|c|c|}
\hline \multirow[t]{2}{*}{ Parameter } & & \multicolumn{2}{|c|}{$\mathrm{HCC}$ in $\mathrm{NCL} ; \mathrm{n}=93$} & \multicolumn{2}{|c|}{ HCC in LC; $n=571$} & \multirow[t]{2}{*}{$p$} \\
\hline & & n/median & $\% /$ range & $\mathrm{n} /$ median & $\% /$ range & \\
\hline \multirow[t]{2}{*}{ Gender } & Male & 68 & 73 & 468 & 82 & 0.045 \\
\hline & Female & 25 & 27 & 103 & 18 & \\
\hline Age & & 69 & $32-85$ & 66 & $31-85$ & 0.004 \\
\hline $\mathrm{BMI}$ & & 24.68 & 19.18-38.5 & 27.1 & $18.07-46.30$ & $<0.001$ \\
\hline \multirow[t]{5}{*}{ ECOG status } & & $n=79$ & & $n=428$ & & 0.432 \\
\hline & 0 & 37 & 46.84 & 195 & 45.56 & \\
\hline & 1 & 30 & 37.97 & 148 & 34.58 & \\
\hline & 2 & 12 & 15.19 & 72 & 16.82 & \\
\hline & 3 or 4 & 0 & 0 & 13 & 3.04 & \\
\hline Diabetes mellitus & Yes & $42(n=59)$ & 71.2 & $308(n=368)$ & 83.7 & 0.020 \\
\hline History of nicotine abuse & Yes & $22(n=55)$ & 40.0 & $110(n=231)$ & 47.6 & 0.308 \\
\hline \multirow[t]{5}{*}{ Etiology } & Alcohol & 14 & 15.05 & 285 & 49.91 & 0.008 \\
\hline & Viral & 7 & 7.53 & 91 & 15.94 & \\
\hline & NAFLD & 6 & 6.45 & 37 & 6.48 & \\
\hline & Other & 13 & 13.98 & 93 & 16.29 & \\
\hline & Unknown & 53 & 56.99 & 65 & 11.38 & \\
\hline
\end{tabular}






Figure 1 Age distribution of patients with HCC in NCL and LC at initial diagnosis.

chronic liver disease was identified, but in this subgroup of patients the prevalence of metabolic risk factors was also high: $37.74 \%$ suffered from diabetes mellitus, $58.5 \%$ from arterial hypertension and $22.64 \%$ presented with a BMI $\geq 25 \mathrm{~kg} / \mathrm{m}^{2}$.

A histopathological report regarding the tumor tissue was available in $87(93.5 \%)$ patients. In 21 patients with HCC in NCL the histopathological evaluation of nonneoplastic liver tissue revealed fibrosis of different degrees, in 18 patients fatty liver disease was diagnosed, in 8 patients no fibrosis was detected in non-neoplastic liver tissue.

A comparison of tumor stage at diagnosis revealed that patients with $\mathrm{HCC}$ in NCL were diagnosed in more advanced tumor stages than patients with HCC in LC. More than half of the patients with HCC in NCL were in BCLC stage C (51.6\% compared to $42.4 \%$ of patients with $\mathrm{HCC}$ in LC), every third patient was diagnosed in intermediate stage (BCLC B, 34.4\% in comparison to $29.6 \%$ of patients with HCC in LC). Tumor related characteristics of the two cohorts are summarized in Table 2. Although diagnosed more frequently as uninodular disease $(49.46 \%)$ than in patients with $\mathrm{LC}, \mathrm{HCC}$ in NCL were larger than those in $\mathrm{LC}$ and were diagnosed more frequently with extrahepatic metastases. The prevalence of portal vein thrombosis was equal between the two cohorts. Ascites was more frequent in patients with $\mathrm{HCC}$ in LC.

The proportion of patients with serum AFP concentration exceeding $200 \mathrm{ng} / \mathrm{ml}$ was almost similar between the two groups $(33.3 \%$ in NCL vs $37.0 \%$ in LC).
In the laboratory work-up of the two cohorts (Table 3) statistically significant differences were apparent with respect to liver function at time of diagnosis of $\mathrm{HCC}$. Patients with HCC in LC showed statistically significant elevated concentrations of bilirubin, ASAT, GGT and IgA as well as prolonged PTT in comparison to patients with HCC in NCL. Concentrations of parameters mirroring liver synthesis capacity (quick, albumin) and platelets were significantly lower in patients with HCC in LC compared to non-cirrhotic patients. All other parameters evaluated did not show statistically significant differences.

In a univariate Cox regression analysis for factors influencing survival of patients with HCC in NCL the presence of portal vein thrombosis, the presence of extrahepatic metastases, concentration of AFP, ASAT and platelets as well as the ECOG performance status of the patient were significant factors. A multivariate analysis of these factors confirmed the presence of extrahepatic metastases and the concentration of platelets as factors significantly influencing survival of patients with HCC in NCL (Table 4).

The unadjusted results of further univariate explorative analyses are given in Figures 2 (demographic parameters and tumor related parameters) and 3 (staging systems).

Regarding the two staging systems applied, the BCLC system correlated better with the survival of patients with HCC in NCL than the CLIP score (Figure 3).

Overall survival according to tumor stage at diagnosis did not differ significantly between patients with HCC in NCL and patients with HCC in liver cirrhosis. Patients diagnosed in BCLC A showed a median survival of 945 days (SD 17) in case of NCL compared to 951 days (SD 176, 
Table 2 Tumor related characteristics of the two cohorts

\begin{tabular}{|c|c|c|c|c|c|c|}
\hline \multirow[t]{2}{*}{ Parameter } & & \multicolumn{2}{|c|}{$\mathrm{HCC}$ in NCL; $n=93$} & \multicolumn{2}{|c|}{$\mathrm{HCC}$ in LC; $n=571$} & \multirow[t]{2}{*}{$p$} \\
\hline & & $\mathrm{n} /$ median & $\%$ \%/range & $\mathrm{n} /$ median & $\overline{\% / \text { range }}$ & \\
\hline \multirow[t]{4}{*}{ Number of nodules } & & \multicolumn{2}{|c|}{$n=93$} & \multicolumn{2}{|c|}{$n=569$} & 0.053 \\
\hline & 1 & 46 & 49.46 & 213 & 37.43 & \\
\hline & 2 & 5 & 5.38 & 60 & 10.54 & \\
\hline & $\geq 3$ & 42 & 45.16 & 296 & 52.02 & \\
\hline Size of largest nodule $(\mathrm{cm})$ & & 9.1 & $2-20$ & 6.5 & $1-22$ & 0.002 \\
\hline \multirow[t]{4}{*}{ Distribution of nodules } & Left liver lobe & 24 & 25.81 & 184 & 32.22 & 0.123 \\
\hline & Right liver lobe & 34 & 36.56 & 157 & 27.50 & \\
\hline & Both liver lobes & 28 & 30.11 & 207 & 36.25 & \\
\hline & No data & 7 & 7.52 & 23 & 4.03 & \\
\hline \multirow[t]{3}{*}{ Portal vein thrombosis } & & $n=86$ & & $\mathrm{n}=517$ & & 0.732 \\
\hline & Yes & 27 & 31.4 & 172 & 33.27 & \\
\hline & No & 59 & 69.6 & 345 & 66.73 & \\
\hline \multirow[t]{3}{*}{ Extrahepatic metastasis } & & $n=86$ & & $n=508$ & & 0.028 \\
\hline & Yes & 32 & 37.21 & 131 & 25.79 & \\
\hline & No & 54 & 62.79 & 377 & 74.21 & \\
\hline \multirow[t]{3}{*}{ Ascites } & & $n=84$ & & $n=535$ & & $<0.001$ \\
\hline & Yes & 17 & 20.24 & 237 & 44.30 & \\
\hline & no & 67 & 79.76 & 298 & 55.70 & \\
\hline \multirow[t]{5}{*}{ BCLC stage } & & $n=93$ & & $\mathrm{n}=571$ & & 0.006 \\
\hline & A & 13 & 14 & 100 & 17.5 & \\
\hline & B & 32 & 34.4 & 169 & 29.6 & \\
\hline & $C$ & 48 & 51.6 & 242 & 42.4 & \\
\hline & $\mathrm{D}$ & 0 & 0 & 60 & 10.5 & \\
\hline \multirow[t]{8}{*}{ CLIP score } & & $n=82$ & & $n=483$ & & 0.015 \\
\hline & 0 & 14 & 17.07 & 60 & 12.42 & \\
\hline & 1 & 20 & 24.39 & 111 & 22.98 & \\
\hline & 2 & 16 & 19.51 & 103 & 21.33 & \\
\hline & 3 & 25 & 30.49 & 88 & 18.22 & \\
\hline & 4 & 5 & 6.1 & 59 & 12.22 & \\
\hline & 5 & 2 & 2.44 & 52 & 10.77 & \\
\hline & 6 & 0 & 0 & 10 & 2.07 & \\
\hline
\end{tabular}

$\mathrm{p}$-values indicating a statistical significance are printed in bold.

$\mathrm{p}=0.422)$ in LC. Patients in intermediate stage had a median survival of 925 days (SD 189) in comparison to 501 days (SD 58, p =0.100) in case of LC. For patients with advanced disease the median survival was 229 days (SD 34) compared to 209 days (SD 15, $\mathrm{p}=0.438$ ).

First-line treatment modalities applied in patients with HCC in NCL are summarized in Table 5. In summary, $30.12 \%$ of patients underwent treatment in curative intent (resection, liver transplantation or local ablation).

\section{Discussion}

Most of the characteristics reported in the literature over the last decades were confirmed in our analysis, but we also identified some differences compared to published data $[4,11]$.

In $14 \%$ of our study population $\mathrm{HCC}$ has been detected in NCL. Patients with HCC in NCL presented at an older age than patients with HCC in LC and this differs from some of the previous reports $[12,13]$. We confirmed a larger proportion of female patients with HCC in NCL. In both cohorts of patients, with and without LC, more than $50 \%$ of patients initially presented with symptoms and impaired performance status. A previous study from Germany states that $47 \%$ of patients present with tumor symptoms leading to the initial diagnosis of $\mathrm{HCC}$, but $86 \%$ of patients with $\mathrm{HCC}$ in NCL are not restricted in their daily activities at all [5]. 
Table 3 Laboratory work-up of patients with HCC in NCL in comparison to patients with HCC in LC

\begin{tabular}{|c|c|c|c|c|c|c|c|}
\hline \multirow[t]{2}{*}{ Parameter (SI-units) } & \multicolumn{3}{|c|}{ Patients with $\mathrm{HCC}$ in $\mathrm{NCL}$} & \multicolumn{3}{|c|}{ Patients with HCC in LC } & \multirow[t]{2}{*}{$p$} \\
\hline & $n$ & Median & Range & $\mathrm{n}$ & Median & Range & \\
\hline Bilirubin & 88 & 11.5 & $3.3-497.0$ & 547 & 20.6 & $3.3-709.1$ & $<0.001$ \\
\hline Quick & 89 & 97.0 & $18-120$ & 547 & 86.0 & $18-120$ & $<0.001$ \\
\hline Albumin & 81 & 40.10 & $15.6-71.0$ & 507 & 36.1 & $9.5-72.5$ & $<0.001$ \\
\hline ALAT & 87 & 0.75 & $0.04-7.72$ & 545 & 0.77 & $0.12-31.20$ & 0.110 \\
\hline ASAT & 86 & 0.76 & $0.17-12.11$ & 546 & 1.11 & $0.26-25.90$ & $<0.001$ \\
\hline GGT & 80 & 2.43 & $0.19-36.28$ & 518 & 3.26 & $0.17-66.51$ & 0.002 \\
\hline Alcaline phosphatase & 81 & 3.59 & $0.38-36.46$ & 506 & 3.41 & $0.69-138.69$ & 0.737 \\
\hline Creatinine & 87 & 78.00 & $45-480$ & 535 & 77.0 & $2-557$ & 0.622 \\
\hline Urea nitrogen & 83 & 5.8 & $2.1-44.2$ & 513 & 5.9 & $1.6-35.8$ & 0.437 \\
\hline Ferritin & 32 & 452.5 & $21-2928$ & 233 & 356.0 & $3-7585$ & 0.553 \\
\hline Transferrin saturation & 29 & 25.50 & $8.0-95.3$ & 166 & 30.3 & 4.5-119.0 & 0.310 \\
\hline Coeruloplasmin & 16 & 0.38 & $0.29-0.53$ & 112 & 0.36 & $0.12-2.31$ & 0.951 \\
\hline Hemoglobin & 82 & 8.2 & $5.7-18.7$ & 505 & 8.1 & $4.1-13.7$ & 0.997 \\
\hline Platelets & 81 & 261.0 & $84-693$ & 506 & 166.5 & $33-799$ & $<0.001$ \\
\hline PTT & 84 & 28.5 & $22.1-60.0$ & 527 & 31.5 & $19.2-80.2$ & $<0.001$ \\
\hline $\lg A$ & 15 & 2.56 & $0.61-22.80$ & 101 & 5.42 & $1.27-43.30$ & $<0.001$ \\
\hline $\lg M$ & 13 & 0.970 & $0.5-2.6$ & 96 & 1.19 & $0.2-15.5$ & 0.274 \\
\hline $\lg G$ & 14 & 13.05 & $8-28$ & 103 & 16.30 & $2-46$ & 0.117 \\
\hline CA 19-9 & 22 & 35.5 & $1.5-7520$ & 226 & 60.00 & $<0.5-8483.0$ & 0.057 \\
\hline AFP & 85 & 32.10 & $1-378813.00$ & 531 & 62.00 & $0.6-1460000.00$ & 0.168 \\
\hline
\end{tabular}

ALAT = alanine aminotransferase, ASAT = aspartate aminotransferase, GGT = gamma glutamyl transferase, $\mathrm{PPT}=$ partial thromboplastin time, IgA = immunoglobulin A, IgG = immunoglobulin G, AFP = alpha fetoprotein, CA 19-9= carbohydrate antigen 19-9. p-values indicating a statistical significance are printed in bold.

For the majority of patients with HCC in NCL the etiology was most likely related to the metabolic syndrome. Even though non-alcoholic fatty liver disease (NAFLD) was diagnosed in a small proportion of patients, factors of the metabolic syndrome were frequent in our cohort, especially in the subgroup of patients in whom no other risk factor was identified. NAFLD, the hepatic manifestation of the metabolic syndrome, is frequently underdiagnosed in the general population but is recognized to be a frequent cause for HCC [14]. The incidence of cirrhosis, on the other hand, in patients with HCC and NAFLD is low in comparison to patients with other causes of HCC [15], and patients with HCC and NAFLD exhibit more features of the metabolic syndrome [15]. Vice versa, about $75 \%$ of obese patients suffer from NAFLD [16], and obesity alone has been shown to be a risk factor for HCC with an OR of 1.39 to 4.52 [17-19].

Several cohort studies have demonstrated that diabetes mellitus is an independent risk factor for hepatocellular carcinoma [20]. An analysis within the SEER-database found a 2.9 fold risk for diabetic patients to develop HCC [21]. This risk factor was present in more than $70 \%$ of our patients with HCC in NCL.
It is suggested that tumor suppressor genes play an important role in the development of steatosis, induce liver cell damage and therefore promote the formation of HCC in the absence of cirrhosis $[15,22,23]$ in combination with other complex dysregulated mechanisms and pathways in fatty liver disease.

It is striking that, although a direct hepatocarcinogenic role of alcohol has not been proven so far, $15 \%$ of patients with HCC in NCL reported a significant intake of alcohol as risk factor for liver disease. This goes along with data previously reported in which $12 \%$ to $21.4 \%$ of patients with HCC in NCL abused alcohol $[4,5,7]$. This suggests that cirrhosis is not a condition sine qua non in alcoholic liver disease for HCC development. It is not known whether in these patients the inflammation alone is sufficient or whether fibrosis needs to develop before HCC can arise. A Swedish study demonstrated a 2.4 fold risk for hepatocellular carcinoma in patients with alcohol abuse in comparison to the general population that increased to a 22.4 fold risk in the presence of cirrhosis [24]. An interaction between alcoholic liver disease and other risk factors is likely to occur. Hence, obesity and the metabolic syndrome are factors which favor the 
Table 4 Cox regression analysis for factors influencing survival in patients with HCC in NCL

\begin{tabular}{|c|c|c|c|c|c|c|}
\hline \multirow{2}{*}{$\begin{array}{l}\text { Variables (SI-units if not indicated } \\
\text { otherwise) }\end{array}$} & \multicolumn{3}{|c|}{ Univariate analysis } & \multicolumn{3}{|c|}{ Multivariate analysis } \\
\hline & HR & $95 \% \mathrm{Cl}$ & $p$ & HR & $95 \% \mathrm{Cl}$ & $p$ \\
\hline Age ( $\leq 65$ years vs $>65$ years) & 1.598 & $0.957-2.669$ & 0.073 & & & \\
\hline Sex (m vs f) & 0.777 & $0.457-1.332$ & 0.352 & & & \\
\hline $\mathrm{BMI}\left(\leq 30 \mathrm{~kg} / \mathrm{m}^{2}\right.$ vs $\left.>30 \mathrm{~kg} / \mathrm{m}^{2}\right)$ & 0.573 & $0.216-1.518$ & 0.263 & & & \\
\hline Etiology (alcohol vs viral vs other) & & & 0.095 & & & \\
\hline BCLC stage & & & 0.014 & & & \\
\hline CLIP & & & 0.031 & & & \\
\hline Size of largest nodule (>5 cm vs $\leq 5 \mathrm{~cm}$ ) & 1.991 & 0.993-3.992 & 0.052 & & & \\
\hline Portal vein thrombosis (yes vs no) & 1.859 & $1.066-3.241$ & 0.029 & 1.559 & $0.676-3.596$ & 0.298 \\
\hline Extrahepatic metastasis (yes vs no) & 2.104 & $1.248-3.549$ & 0.005 & 2.844 & $1.098-7.368$ & 0.031 \\
\hline Ascites & 1.181 & $0.575-2.428$ & 0.651 & & & \\
\hline Number of lesions (one vs two vs multinodular) & & & 0.204 & & & \\
\hline Steatosis (yes vs no) & 0.576 & $0.248-1.340$ & 0.200 & & & \\
\hline AFP & 1.0 & $0.999-1.000$ & 0.031 & 1.0 & $0.999-1.001$ & 0.323 \\
\hline CA 19-9 & 1.0 & $0.999-1.001$ & 0.969 & & & \\
\hline Hemoglobin & 0.963 & $0.790-1.174$ & 0.709 & & & \\
\hline ALAT & 1.060 & $0.864-1.302$ & 0.575 & & & \\
\hline ASAT & 1.158 & $1.000-1.340$ & 0.050 & 0.999 & $0.629-1.586$ & 0.997 \\
\hline Albumin & 0.975 & $0.941-1.010$ & 0.152 & & & \\
\hline Bilirubin & 1.001 & $0.997-1.006$ & 0.484 & & & \\
\hline Alcaline phosphatase & 0.993 & $0.955-1.032$ & 0.724 & & & \\
\hline GGT & 1.021 & $0.974-1.071$ & 0.386 & & & \\
\hline Quick & 0.990 & $0.977-1.002$ & 0.112 & & & \\
\hline PTT & 1.057 & $1.007-1.110$ & 0.026 & 1.057 & $0.891-1.255$ & 0.524 \\
\hline Platelets & 1.002 & $1.000-1.004$ & 0.049 & 1.004 & $1.001-1.007$ & 0.006 \\
\hline Ferritin & 1.0 & $1.000-1.001$ & 0.455 & & & \\
\hline Creatinine & 0.997 & $0.992-1.002$ & 0.231 & & & \\
\hline Urea nitrogen & 0.966 & $0.914-1.020$ & 0.212 & & & \\
\hline Diabetes mellitus (yes vs no) & 1.246 & $0.564-2.753$ & 0.586 & & & \\
\hline Nicotine (yes vs no) & 1.239 & $0.632-2.428$ & 0.532 & & & \\
\hline ECOG status ( 1 or 2 vs 0 ) & 1.965 & $1.129-3.417$ & 0.017 & 1.645 & $0.681-3.968$ & 0.269 \\
\hline Aim of treatment (palliative vs curative) & 1.044 & $0.616-1.770$ & 0.873 & & & \\
\hline
\end{tabular}

p-values indicating a statistical significance are printed in bold.

progression of alcoholic liver disease and increase hepatocellular carcinoma (HCC) incidence and mortality [25]. Similarly, a synergism has been shown for viral hepatitis and heavy alcohol consumption [26]. Viral hepatitis played a minor role in our cohort.

Since we did not take biopsies from non-tumorous liver tissue as part of the standard work-up of patients with HCC, the number of patients with histology from non-tumorous tissue is rather small. Patients without any hepatic damage of the tissue surrounding the tumor were few and this may indicate the possibility of direct hepatocarcinogenesis in the healthy liver (e.g. adenomacarcinoma sequence). Most patients showed histological features of chronic liver damage. Chronic liver disease thus may not have been recognized in advance of hepatocellular carcinoma and therefore no surveillance has been offered. This is probably the reason why diagnosis is made in advanced tumor stages in these patients.

Consistent with previous studies almost every second patient with HCC in NCL was diagnosed with a single tumorous nodule but of larger size [11,27]. The proportion of patients with extrahepatic metastases in our cohort exceeded $25 \%$ and was strikingly larger than reported in other cohorts $[5,28]$ with up to $15 \%$.

The BCLC staging system, although developed for patients with LC, correlates with patients' survival. The 


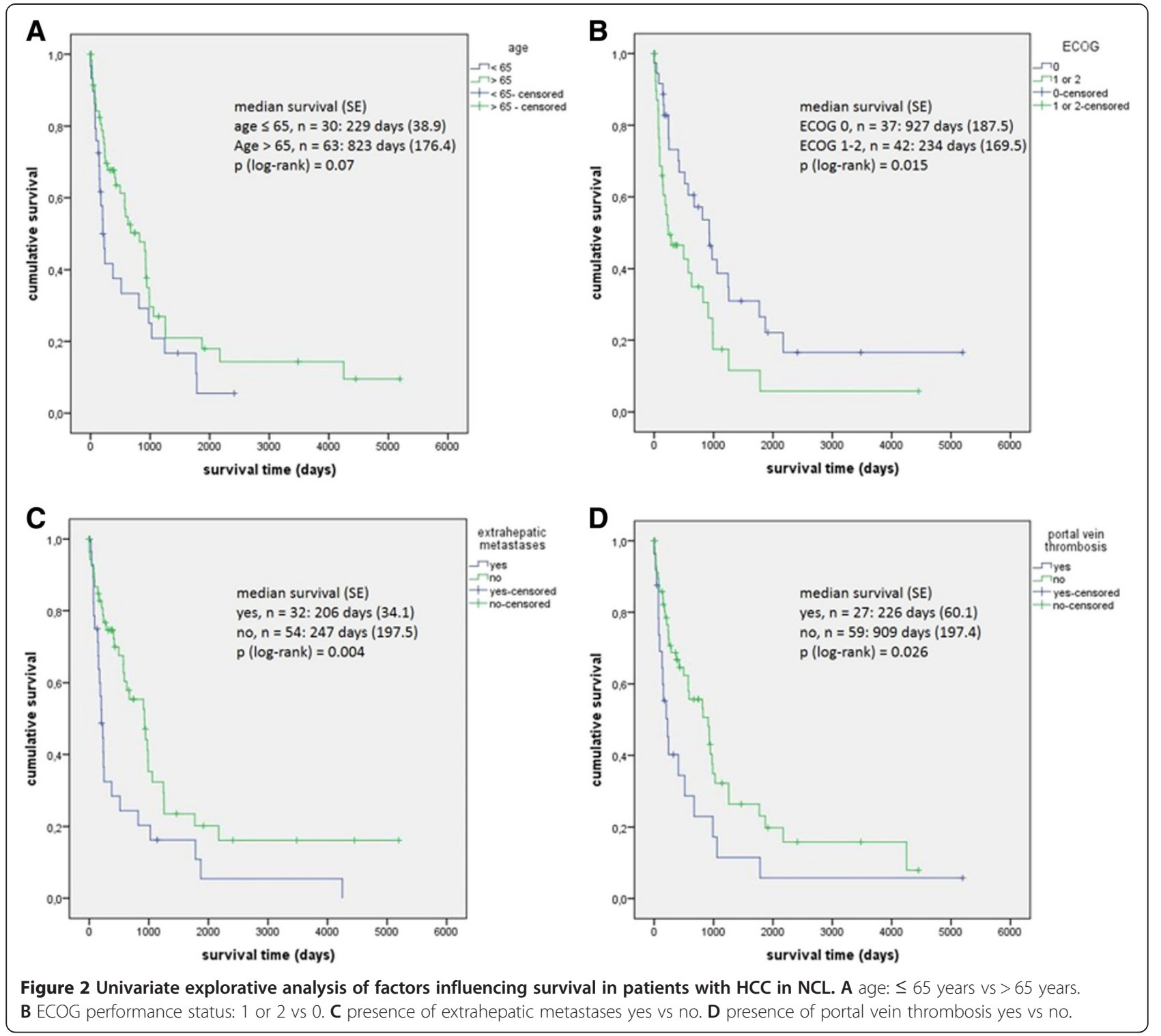

CLIP score was less suitable to predict survival of patients with HCC in NCL. Patients in intermediate stage HCC according to BCLC in NCL show a trend towards prolonged survival in comparison to patients with LC. This is most likely because curative treatment can be offered to these patients even if the tumor load exceeds the Milan criteria.

Survival of patients with HCC in NCL mainly depends on tumor related factors such as tumor size, existence of satellite lesions, existence of a tumor capsule, vascular invasion, grading, $\mathrm{R} 0$ resection and the amount of intraoperative blood transfusions [28-35]. Analyses from mixed surgically and medically treated cohorts of patients identified tobacco consumption, clinical performance status, siderosis of non-tumorous tissue, tumor stage according to $\mathrm{BCLC}$ and the initial treatment to be relevant for survival [5,7]. Our study confirms tumor related factors to exert a significant influence on patients' outcome in univariate explorative analyses (portal vein thrombosis, existence of extrahepatic tumor manifestations). Tumor size and number of intrahepatic HCC nodules had not statistically significant impact on survival. Ongoing hepatic inflammation, mirrored in elevation of ASAT, reduced hepatic synthesis capacity mirrored in prolonged PTT and low platelets (probably a consequence of portal hypertension caused by portal vein thrombosis or by large hepatic tumor load) were negative predictive for survival for patients with HCC in NCL. At multivariate analysis solely the presence of metastases and low platelets were significant factors influencing survival.

Although the proportion of patients who presented at an early tumor stage was rather small, it was evident that 

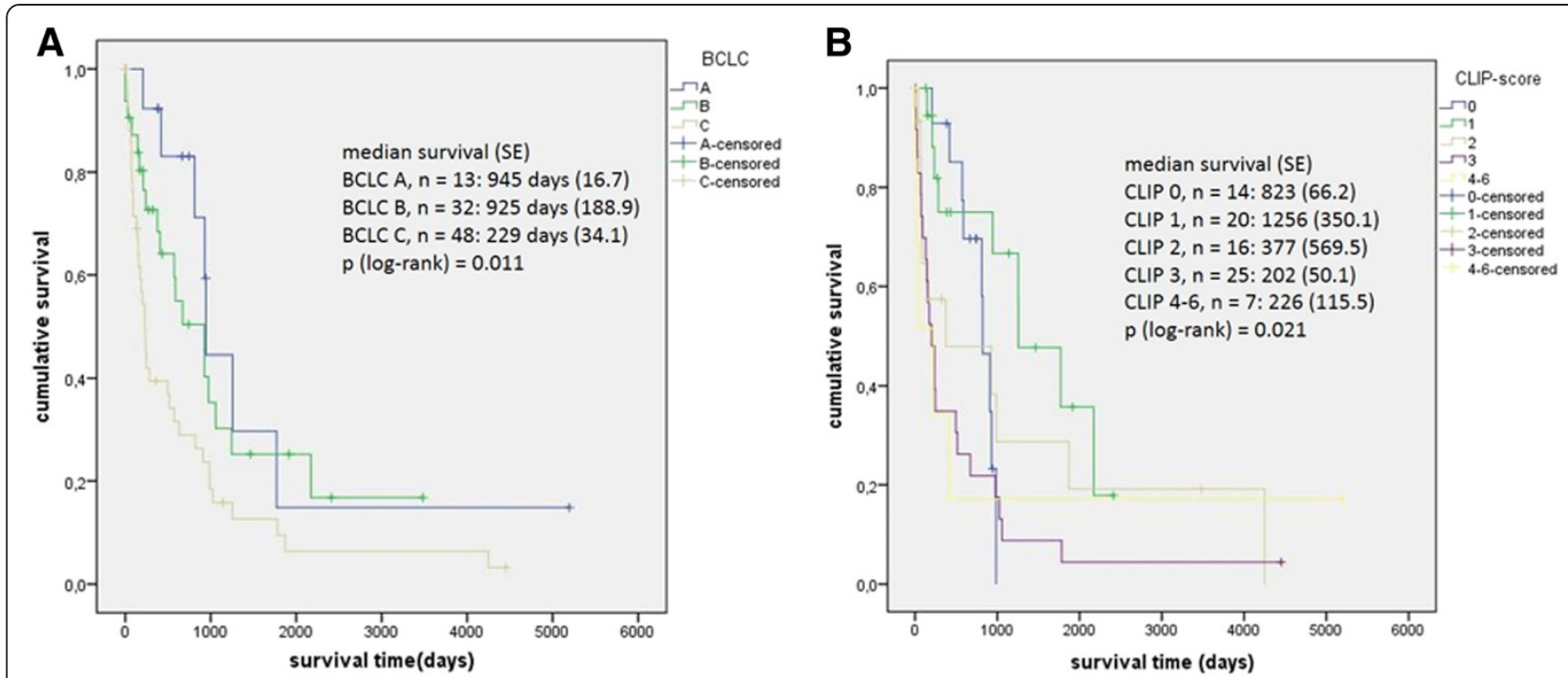

Figure 3 Univariate explorative analyses of survival of patients with HCC in NCL in dependence of tumor stage according to BCLC and CLIP-score. A survival (days) according to BCLC score A vs B vs C. B survival (days) according to CLIP score 0 vs 1 vs 2 vs 3 vs 4-6.

in addition to patients in BCLC A some patients at an intermediate tumor stage (BCLC B) also qualified for treatment in curative intent. A modification of current treatment algorithms for patients with HCC in NCL is desirable.

Our study has some limitations due to the retrospective design and the small number of patients in some of the univariate analyses might lead to insignificant findings in the statistical analyses. The mixed cohort of patients does not permit to address surgical aspects.

Table 5 Treatment modalities applied in patients with HCC in NCL $(n=93)$ and patients with HCC in LC $(n=571)$

\begin{tabular}{|c|c|c|c|c|c|}
\hline & \multirow[b]{2}{*}{ Treatment modality } & \multicolumn{2}{|c|}{$\begin{array}{l}\mathrm{HCC} \text { in } \\
\mathrm{NCL}\end{array}$} & \multicolumn{2}{|c|}{$\begin{array}{l}\text { HCC in } \\
\text { LC }\end{array}$} \\
\hline & & $\bar{n}$ & $\%$ & $n$ & $\%$ \\
\hline \multirow[t]{3}{*}{ Curative treatment } & Resection & 22 & 23.66 & 69 & 12.1 \\
\hline & RFA/thermoablation & 5 & 5.38 & 27 & 4.7 \\
\hline & Liver transplantation & 1 & 1.08 & 8 & 1.4 \\
\hline Total & & 28 & 30.11 & 104 & 18.2 \\
\hline \multirow[t]{5}{*}{ Palliative treatment } & $\begin{array}{l}\text { Transarterial } \\
\text { chemoembolization }\end{array}$ & 14 & 15.05 & 128 & 22.4 \\
\hline & $\begin{array}{l}\text { Systemic therapy } \\
\text { (chemotherapy } \\
\text { or sorafenib) }\end{array}$ & 22 & 23.66 & 167 & 29.3 \\
\hline & Y90-radioembolisation & 3 & 3.23 & 23 & 4.0 \\
\hline & $\begin{array}{l}\text { HD-interstitial } \\
\text { brachytherapy }\end{array}$ & 12 & 12.90 & 52 & 9.1 \\
\hline & Best supportive care & 5 & 5.38 & 20 & 3.5 \\
\hline \multirow[t]{2}{*}{ Total } & & 56 & 60.22 & 390 & 68.3 \\
\hline & No data & 9 & 9.68 & 77 & 13.5 \\
\hline
\end{tabular}

\section{Conclusion}

HCC in NCL has distinct features compared to HCC in LC. The majority of patients with HCC in NCL present with signs of hepatic damage even in the absence of LC. Risk factors for LC and risk factors for NAFLD are present in the majority of these patients and surveillance programs need to be implemented if these are cost-effective.

In this cohort the proportion of patients with advanced tumor stage and especially with extrahepatic metastases was larger than expected from previously published surgically treated cohorts. The BCLC staging algorithm correlates with patients' survival. However, therapeutic decisions should not be based on this staging system as in non-cirrhotic patients curative treatment can be offered also to patients with large intrahepatic tumors outside the Milan criteria.

\section{Competing interests}

This work was not supported by any grant or funding source. The authors disclose no potential competing interests.

\section{Authors' contributions}

$K S, C S, J P, J B, T B$, JA performed the study. KS and KA performed the statistical analysis. KS, CS and JB wrote the paper. PM and JR supervised and drafted the study and the manuscript and performed a final revision. All authors read and approved the final manuscript.

\section{Acknowledgement}

The manuscript contains parts of the medical thesis of Janine Poranzke. Parts of this work were presented at the UEGW 2013 (P1195) and published in abstract form.

\section{Author details}

'Department of Gastroenterology, Hepatology and Infectious Diseases, Otto-von-Guericke University Magdeburg, Leipziger Str. 44, Magdeburg 39120, Germany. ${ }^{2}$ Institute for Biometry and Medical Informatics,

Otto-von-Guericke University, Magdeburg, Germany. ${ }^{3}$ Department of 
Radiology and Nuclear Medicine, Otto-von-Guericke University, Magdeburg, Germany. ${ }^{4}$ Department of General, Visceral and Vascular Surgery,

Otto-von-Guericke University, Magdeburg, Germany.

Received: 23 February 2014 Accepted: 26 June 2014

Published: 3 July 2014

\section{References}

1. El-Serag HB: Hepatocellular carcinoma. N Engl J Med 2011, 365(12):1118-1127.

2. Schütte K, Bornschein J, Malfertheiner P: Hepatocellular carcinomaepidemiological trends and risk factors. Dig Dis 2009, 27(2):80-92.

3. Globocan. In 2008. http://globocan.iarc.fr/.

4. Trevisani F, Frigerio M, Santi V, Grignaschi A, Bernardi M: Hepatocellular carcinoma in non-cirrhotic liver: a reappraisal. Dig Liver Dis 2010, 42(5):341-347.

5. Wörns MA, Bosslet T, Victor A, Koch S, Hoppe-Lotichius M, Heise M, Hansen T, Pitton MB, Niederle IM, Schuchmann M, Weinmann A, Düber C, Galle PR, Otto G: Prognostic factors and outcomes of patients with hepatocellular carcinoma in non-cirrhotic liver. Scand J Gastroenterol 2012, 47(6):718-728.

6. Schütte K, Kipper M, Kahl S, Bornschein J, Götze T, Adolf D, Arend J, Seidensticker R, Lippert H, Ricke J, Malfertheiner P: Clinical characteristics and time trends in etiology of hepatocellular cancer in Germany. Digestion 2013, 87(3):147-159.

7. Giannini EG, Marenco S, Bruzzone L, Savarino V, Farinati F, Del Poggio P, Rapaccini GL, Di Nolfo MA, Benvegnù L, Zoli M, Borzio F, Caturelli E, Chiaramonte $M$, Trevisani F: Hepatocellular carcinoma in patients without cirrhosis in Italy. Dig Liver Dis 2013, 45(2):164-169.

8. Llovet JM, Brú C, Bruix J: Prognosis of hepatocellular carcinoma: the BCLC staging classification. Semin Liver Dis 1999, 19(3):329-338.

9. CLIP investigator group: Prospective validation of the CLIP score: a new prognostic system for patients with cirrhosis and hepatocellular carcinoma. The Cancer of the Liver Italian Program (CLIP) Investigators. Hepatology 2000, 31(4):840-845

10. Grieco A, Pompili M, Caminiti G, Miele L, Covino M, Alfei B, Rapaccini GL, Gasbarrini G: Prognostic factors for survival in patients with earlyintermediate hepatocellular carcinoma undergoing non-surgical therapy: comparison of Okuda, CLIP, and BCLC staging systems in a single Italian centre. Gut 2005, 54(3):411-418.

11. Alkofer B, Lepennec $V$, Chiche L: Hepatocellular cancer in the non-cirrhotic liver. J Visc Surg 2011, 148(1):3-11.

12. Trevisani F, D'Intino PE, Caraceni P, Pizzo M, Stefanini GF, Mazziotti A Grazi GL, Gozzetti G, Gasbarrini G, Bernardi M: Etiologic factors and clinical presentation of hepatocellular carcinoma. Differences between cirrhotic and noncirrhotic Italian patients. Cancer 1995, 75(9):2220-2232.

13. Chang C, Chau G, Lui W, Tsay S, King K, Wu C: Long-term results of hepatic resection for hepatocellular carcinoma originating from the noncirrhotic liver. Arch Surg 2004, 139(3):320-5. discussion 326.

14. Baffy G, Brunt EM, Caldwell SH: Hepatocellular carcinoma in non-alcoholic fatty liver disease: an emerging menace. J Hepatol 2012, 56(6):1384-1391.

15. Ertle J, Dechêne A, Sowa J, Penndorf V, Herzer K, Kaiser G, Schlaak JF, Gerken G, Syn W, Canbay A: Non-alcoholic fatty liver disease progresses to hepatocellular carcinoma in the absence of apparent cirrhosis. Int J Cancer 2011, 128(10):2436-2443.

16. Rahman R, Hammoud GM, Almashhrawi AA, Ahmed KT, Ibdah JA: Primary hepatocellular carcinoma and metabolic syndrome: An update. World J Gastrointest Oncol 2013, 5(9):186-194.

17. Caldwell SH, Crespo DM, Kang HS, Al-Osaimi, Abdullah MS: Obesity and hepatocellular carcinoma. Gastroenterology 2004, 127(5 Suppl 1):S97-103.

18. Borena W, Strohmaier S, Lukanova A, Bjørge T, Lindkvist B, Hallmans G, Edlinger M, Stocks T, Nagel G, Manjer J, Engeland A, Selmer R, Häggström C, Tretli S, Concin H, Jonsson H, Stattin P, Ulmer H: Metabolic risk factors and primary liver cancer in a prospective study of 578,700 adults. Int $J$ Cancer 2012, 131(1):193-200.

19. Calle EE, Rodriguez C, Walker-Thurmond K, Thun MJ: Overweight, obesity, and mortality from cancer in a prospectively studied cohort of U.S. adults. N Engl J Med 2003, 348(17):1625-1638.

20. Rosmorduc O, Fartoux L: HCC and NASH: how strong is the clinical demonstration? Clin Res Hepatol Gastroenterol 2012, 36(3):202-208.

21. Welzel TM, Graubard BI, Zeuzem S, El-Serag HB, Davila JA, McGlynn KA: Metabolic syndrome increases the risk of primary liver cancer in the
United States: a study in the SEER-Medicare database. Hepatology 2011, 54(2):463-471

22. Herzer K, Hofmann TG, Teufel A, Schimanski CC, Moehler M, Kanzler S, Schulze-Bergkamen H, Galle PR: IFN-alpha-induced apoptosis in hepatocellular carcinoma involves promyelocytic leukemia protein and TRAIL independently of p53. Cancer Res 2009, 69(3):855-862.

23. Cazanave SC, Mott JL, Elmi NA, Bronk SF, Werneburg NW, Akazawa Y, Kahraman A, Garrison SP, Zambetti GP, Charlton MR, Gores GJ: JNK1-dependent PUMA expression contributes to hepatocyte lipoapoptosis. J Biol Chem 2009, 284(39):26591-26602.

24. Kuper H, Ye W, Broomé U, Romelsjö A, Mucci LA, Ekbom A, Adami HO, Trichopoulos D, Nyrén O: The risk of liver and bile duct cancer in patients with chronic viral hepatitis, alcoholism, or cirrhosis. Hepatology 2001, 34(4 Pt 1):714-718.

25. Chiang DJ, McCullough AJ: The impact of obesity and metabolic syndrome on alcoholic liver disease. Clin Liver Dis 2014, 18(1):157-163.

26. Yuan J, Govindarajan S, Arakawa K, Yu MC: Synergism of alcohol, diabetes, and viral hepatitis on the risk of hepatocellular carcinoma in blacks and whites in the U.S. Cancer 2004, 101(5):1009-1017.

27. Sarpel U, Ayo D, Lobach I, Xu R, Newman E: Inverse relationship between cirrhosis and massive tumours in hepatocellular carcinoma. HPB (Oxford) 2012, 14(11):741-745.

28. Arnaoutakis DJ, Mavros MN, Shen F, Alexandrescu S, Firoozmand A, Popescu I, Weiss M, Wolfgang CL, Choti MA, Pawlik TM: Recurrence patterns and prognostic factors in patients with hepatocellular carcinoma in noncirrhotic liver: a multi-institutional analysis. Ann Surg Oncol 2014 21(1):147-154.

29. Bège T, Le Treut YP, Hardwigsen J, Ananian P, Richa H, Campan P, Garcia S: Prognostic factors after resection for hepatocellular carcinoma in nonfibrotic or moderately fibrotic liver. A 116-case European series. J Gastrointest Surg 2007, 11(5):619-625.

30. Dupont-Bierre E, Compagnon P, Raoul J, Fayet G, de Lajarte-Thirouard A, Boudjema K: Resection of hepatocellular carcinoma in noncirrhotic liver: analysis of risk factors for survival. J Am Coll Surg 2005, 201(5):663-670.

31. Lang H, Sotiropoulos GC, Brokalaki El, Schmitz KJ, Bertona C, Meyer G, Frilling A, Paul A, Malagó M, Broelsch CE: Survival and recurrence rates after resection for hepatocellular carcinoma in noncirrhotic livers. J Am Coll Surg 2007, 205(1):27-36.

32. Laurent C, Blanc JF, Nobili S, Sa Cunha A, Le Bail B, Bioulac-Sage P, Balabaud C, Capdepont M, Saric J: Prognostic factors and longterm survival after hepatic resection for hepatocellular carcinoma originating from noncirrhotic liver. J Am Coll Surg 2005, 201(5):656-662.

33. Capussotti L, Muratore A, Amisano M, Massucco P, Polastri R, Bouzari H: Liver resection for large-size hepatocellular carcinomas in 47 non-cirrhotic patients-no mortality and long-term survival. Hepatogastroenterol 2006, 53(71):768-772.

34. Grazi GL, Cescon M, Ravaioli M, Ercolani G, Gardini A, Del Gaudio M, Vetrone $G$, Cavallari A: Liver resection for hepatocellular carcinoma in cirrhotics and noncirrhotics. Evaluation of clinicopathologic features and comparison of risk factors for long-term survival and tumour recurrence in a single centre. Aliment Pharmacol Ther 2003, 17 Suppl 2:119-129.

35. Faber W, Sharafi S, Stockmann M, Denecke T, Sinn B, Puhl G, Bahra M, Malinowski MB, Neuhaus $P$, Seehofer D: Long-term results of liver resection for hepatocellular carcinoma in noncirrhotic liver. Surgery 2013 153(4):510-517.

doi:10.1186/1471-230X-14-117

Cite this article as: Schütte et al:: Characterization and prognosis of patients with hepatocellular carcinoma (HCC) in the non-cirrhotic liver. BMC Gastroenterology 2014 14:117. 